obtained from certain seaweeds in South Africa, New Zealand and in Britain. This is being further explored.

To obtain a pure agar, the freshly collected algæ are bleached in the sun for some days, the process being hastened by sprinkling at intervals with fresh water. The bleached algæe are then boiled with water (Japan) or treated with steam (United States) and the resulting mass strained through cloth. The clear liquid is poured into shallow troughs to set, and the jelly cut into narrow strips. In Japan, the process is carried out in cold weather, so that the strips contract, expelling water ; in the United States the same result is obtained by artificial refrigeration. In either case, the expelled water is drained off, and the remaining jelly dried and shredded for packing.

Agar prepared in this manner has been analysed and is described as "a galactan with sugar units of unusual structure". Carrageen mucilage from Chrondrus crispus is a "polysaccharide ester of sulphuric acild by the acetolysis of which two galactans were isolated". Both these products are water soluble. According to Tschudy and Sargent, the two American species of Gigartina require a preliminary soaking and subsequent boiling in lime water or 2 per cent calcium chloride in order to extract a substance giving a firm jelly. Subsequent treatment (straining, cooling and drying) appears to be the same as for the water-soluble derivatives of other types. It appears, therefore, that a number of different but probably closely related substances with similar physical characteristics are included under the general term agar. The amount which can be extracted appears to be very variable. One of the best sources, the Japanese Gelidium amansii, is said to yield 25-35 per cent of its dry weight as agar.

The commercial utilization of seaweeds raises many issues. Having regard to present difficulties of overseas transport, it would seem natural to seek supplies from our own shores, possibly from the west and south-west of England, and the west and north-west of Scotland. The shores must be far enough from towns to escape serious pollution from sewage, yet sufficiently. accessible to the collector and to transport for the material collected. For most purposes, probably, the dried seaweed would suffice, and it could be washed and dried on the spot, before packirg.

When harvesting is regularly practised, it may be necessary to restrict the season of cutting and to regulate it in such a way that regeneration may occur by natural means. Much more information is needed as to the requirements of seaweeds for healthy growth and the reasons underlying the present distribution, especially of useful types. It is very probable that advances along these lines would lead to successful cultivation of desirable types like Gracilaria confervoides, Ahnfelditia plicata or Gelidium corneum, which are fairly common in some localities, or of Chondrus crispus and Gigartina stellata Batt., which are already known to be abundant in certain areas.

The commercial utilization further demands much work on the biochemical and technical side before the final stages of marketing can be reached. We thus require the services of the botanist, the ecologist, the biochemist, the technician and the financier. Yet the last word remains with the botanist, who com. bines the experience and judgment of a systematist with the prictical knowledge of the ecologist. For, in order that analyses may bear fruit, it is necessary that the right type of alga should be recognizable and recognized, its course of life understood and its reproductive periods utilized to the full.

\section{PHYSIOLOGY OF THE LUNG}

\author{
BY DR. O. A. TROWELL, \\ University of Edinburgh
}

$P$ ROF. B. Narayana, in his presidential address to the Physiology Section of the thirtieth Indian Science Congress held in January last, took as his subject "The Growth of Physiology as an Experimental Science". After tracing the history of experimental physiology from its origins with Ludwig and Claude Bernard, and giving some account of its current status, he proceeded to a review of recent work on the vasomotor and bronchomotor mechanisms of the lung, which subject he chose as an illustration of modern methods and the modern outlook in experimental physiology. This field of research has been developed chiefly by Prof. de Burgh Daly and his colleagues, first in Birmingham and later in Edinburgh. Prof. Narayana himself collaborated in some of the work in Edinburgh, and later he and his colleagues in India have made further contributions of their own.

In considering the physiological activity of the lung itself, we may remark that its respiratory movements are merely a passive response to changes in the capacity of the chest, that the gas exchange between blood and air is simply a process of physical diffusion, and that the only known physiological variables intrinsic in the lung itself are the calibre of the blood vessels and the calibre of the bronchi. The calibre of these tubes is controlled by the plain muscle fibres which form a large part of their walls. Vasomotor and bronchomotor changes, therefore, seem to be the principal intrinsic activities of the lung which call for investigation, and this is the field which Daly and his colleagues have successfully explored.

In the physiological investigation of any bodily organ there are, broadly speaking, two alternative lines of attack available. The first, or analytical, method seeks to isolate the organ in question from the disturbing influences of the rest of the body, preferably by excising it altogether, and, keeping it alive under fully controlled but somewhat artificial conditions, to analyse its functional responses to controlled stimuli. The second, or synthetic, method seeks to establish the part played by the organ in the integrated life of the whole animal, how it influences, and is influenced by, changes occurring elsewhere in the body. In the first method natural conditions must be sacrificed in order to secure full experimental control, and the results obtained indicate the physiological potentialities of the organ rather than its normal behaviour in the whole animal. The second method sacrifices full experimental control in order to maintain a natural condition of the organ; the results generally lack the scientific certitude associated with the former method, but when significant they reveal more truly the role of the organ in the normal life of the animal.

Daly, in his work on the lung, adopted the analytical approach. His success has been due in the first place to the development of a skilful technique whereby the lungs can be removed from the body and kept alive for several hours under artificial conditions, the blood circulation and respiratory movements being maintained by suitable pumps. The lung inflation, blood pressure, blood flow and other factors were under complete control and were recorded continuously. A second factor, no less 
essential to success, arose from an anatomical consideration. It was known that in addition to their main supply from the pulmonary artery, the lungs also receive a small vascular supply from the systemic circulation by way of the bronchial arteries. These vessels supply the walls of the bronchi and those of the pulmonary arteries together with their associated nerves and ganglia. In preliminary work, Daly traced the territorial distribution of the bronchial vascular system in the lung; he showed that there is a capillary anastomosis between the bronchial and the pulmonary circulations in the region of the respiratory bronchioles, and he was the first to realize the physiological importance of the fact that the arterial and bronchial musculature, with their associated nerves, the very tissues the responses of which were to be the subject of investigation, received their oxygen supply and nourishment exclusively from the bronchial circulation. $\mathrm{He}$ therefore devised a method for the separate perfusion of the bronchial circulation as well as the pulmonary circulation in the isolated lung. Previous workers on the isolated lung had perfused only the pulmonary vessels, and their usual failure to obtain vasomotor responses was therefore only to be expected. The technique of the 'isolated perfused lung' had to be perfected before any worthwhile investigations were feasible.

The results obtained have provided convincing evidence of the existence of a nervous vasomotor control of the pulmonary circulation; the earlier evidence had been unsatisfactory. The vasomotor and bronchomotor responses to the stimulation of various nerves and to the injection of various hormones and drugs were fully investigated in several species of animal--guinea pig, cat, dog, pig and monkey. The particular responses obtained varied considerably from species to species and also under varying experimental conditions in any one animal, so much so that no simple or uniform picture emerges. This is characteristic of modern physiology ; the more intensively a phenomenon is investigated the more complex is it revealed to be, and the final picture ever recedes from sight. Every day the apparently simple phenomena of classical physiology grow more and more complex as they are subjected to the more searching analysis of modern technique.

With regard to the vasomotor responses, stimulation of either cholinergic (vagus) fibres or adrenergic (sympathetic) fibres most commonly produced vasoconstriction, but in certain defined circumstances vasodilation could result from either. Injection of acetyl choline or adrenaline gave parallel results. The bronchomotor responses were similarly variable, depending on the species and the experimental conditions. The detailed findings are of considerable interest, particularly in their relation to current theories of chemical transmission in the autonomic system, but space does not permit their discuission here.

Perhaps the most significant outcome of this work is to be found not in the detailed findings, but rather in the very variability of the responses obtained; the fact that a given nerve or a given hormone can produce in certain circumstances a constriction and in other circumstances a dilatation in one and the same preparation is in itself of the greatest physiological importance, and suggests many hitherto unthought-of possibilities with regard to the occurrence of similar changes in the whole animal. The problem of the bronchospasm in asthma, for example, can be considered in quite a new light as a result of these researches. It must always be remembered. however, that the isolated perfused lung is surviving under highly abnormal conditions and probably its behaviour is correspondingly abnormal; so the transference of these results to the whole animal and to man is at present not justified. The potentialities of the lung have been revealed; to what extent they operate in normal life remains to be discovered.

Prof. Narayana concluded his address with a plea for the further development of experimental physiology in India. He himself is already contributing to this end in the active research which he has initiated in his own department at the University of Patna. We can wish our Indian colleagues well in their pursuit of the common ideal, and we shall look forward to their contributions to the further growth of the science.

\section{OBITUARIES}

\section{Mr. William Hartree, O.B.E.}

A REMARKaBle fact about William Hartree, who died on April 27, 1943, was that his first scientific paper appeared (in 1920) when he was fifty, his last (in 1941) when he was seventy-one. In those twentyone years he published ten papers alone and thirtysix in collaboration (in J. Physiol., Proc. Roy. Soc. A and B, Phil. Trans. A and B, Phys. Rev., J. Sci. Instruments, Proc. Camb. Phil. Soc., and Biochem. J.).

In the strictest and best sense, Hartree was an amateur; he worked for love of his work and, I think one dare say, of his collaborators. But he worked also with the intensity and pride of a craftsman in his job, and for all his steadfast modesty, he knew, like a craftsman, when he had done it well, and he worked with quite inflexible devotion. During the War of 1914-18, at Whale Island, he said -almost seriously - that he was going on strike for more work and less pay: his pay could not have been much less nor his work more. I had to stop him then from trying to get into the army at forty-seven -because he was enjoying that work too much! Later, at Cambridge, when I pressed him once to take a holiday, he wrote me next day "I have taken a holiday to-day-made a new kind of experiment"the only kind of holiday he liked.

Hartree was known, except by his work, to very few : he never attended meetings: he wanted no credit or praise for what he did. He almost seemed to prefer to do a difficult job well rather than make it easy. He was ready to face the most laborious calcu. lations in order to obtain a result; he almost resented at first any improvement in technique which made calculation simpler. In his work with me on the physiology of muscle he reckoned that he had written down between $10^{7}$ and $10^{8}$ figures, and we very seldom discovered a mistake. He was always ready to take a sporting chance to see what he could make of a new job-as when he took up research on A.A. gunnery in 1916, when he turned (a mathematician and engineer) to experimental physiological research in 1919 , when he took to the theoretical study of atomic structures in 1933 and to the numerical solution of certain partial differential equations, concerned with motion in a viscous fluid, in his last years. Yet in some ways he was very conservative; for thirteen years his chair in the physiological laboratory at Cambridge, where he did his arithmetic, 